\title{
Solar Radiation Durability Framework Applied To Acrylic Solar Mirrors
}

\author{
Myles P. Murray ${ }^{\mathrm{a}}$, Devin Gordon ${ }^{\mathrm{a}}$, Scott A. Brown ${ }^{\mathrm{b}}$ Wei-Chun Lin ${ }^{\mathrm{a}}$, Kara A. Shell ${ }^{\mathrm{a}}$, Mark A. \\ Schuetz ${ }^{\mathrm{b}}$, Sean Fowler ${ }^{\mathrm{c}}$, Jim Elman ${ }^{\mathrm{d}}$, Roger H. French ${ }^{\mathrm{a}^{*}}$ \\ a Materials Science, 510 White, 10900 Euclid Ave., Case Western Reserve University, Cleveland \\ $\mathrm{OH}$ 44106-7204 \\ b Replex Plastics, 11 Mount Vernon Ave, Mount Vernon, Ohio 43050 \\ c Q-Lab, 800 Canterbury Road, Cleveland, OH 44145 \\ d Filmetrics Inc. 250 Packett's Landing Fairport, NY 14450
}

\begin{abstract}
Mirror augmented photovoltaic (MAPV) systems utilize low cost mirrors to couple more light into a photovoltaic (PV) absorber. By increasing the light absorbed, they are expected to produce less expensive electricity. As a substrate candidate for back surface reflector mirrors, two grades of PMMA have been exposed to UV stress from two sources at two intensities for two doses in an effort to see the response of materials under different states of stress and after exposure to different amounts of total stress. By developing a framework for correlating stresses, such as short wave ultraviolet radiation, with responses, such as induced absorbance and yellowing, mirror durability we have made progress in developing lifetime and degradation science using mirror durability as a case study. All of the samples showed similarities in their degradation characteristics. The UV stress acceleration factor was quantized as 10.2 in short wave ultraviolet irradiance, and 15.8 in total shortwave UV dose. The effects of UV absorbers in protecting the polymer from degradation are discussed. Further study into degradation mechanisms will elucidate the exact phenomena that contribute to these material responses to stress.
\end{abstract}

Keywords: Photovoltaics, Optical Properties, Radiation Durability, Photodegradation, Lifetime, Acrylic, Mirrors

* roger.french@,case.edu, 216-368-3655, http://sdle.case.edu , http://vuv.case.edu

\section{Introduction}

Concentrating Photovoltaic (CPV) systems may present better value than traditional photovoltaic technologies. An example of low concentration PV is Mirror Augmented Photovoltaic (MAPV) systems, which are systems with the potential to reduce the Levelized Cost Of Energy (LCOE) when compared with traditional flat panel PV technologies. In MAPV systems electrical output per unit area of active materials increases nearly linearly with concentration factor, and since simple mirrors can cost $90 \%$ less than a PV system, they present a good value proposition.

The PV industry suffered a catastrophic failure of a MAPV system at Carissa Plains in the 1980s when higher UV exposure, increased operating temperatures, poor process control and harsh climates led to interconnect failure, encapsulant browning, and very rapid power degradation. Due in part to this failure, module manufactures may not honor warranties on PV modules that are used with increased irradiance, rendering MAPV an unviable option.

A recent U. S. Department of Energy workshop on Science for Energy Technologies, ${ }^{1}$ identified the topic of PV lifetime and degradation science (L\&DS) as a critical scientific challenge for robust adoption of PV going

Reliability of Photovoltaic Cells, Modules, Components, and Systems IV, edited by Neelkanth G. Dhere, John H. Wohlgemuth, Kevin W. Lynn, Proc. of SPIE Vol. 8112,

811203 - (c) 2011 SPIE · CCC code: 0277-786X/11/\$18 - doi: 10.1117/12.893827

Proc. of SPIE Vol. $8112811203-1$ 
forward. By applying the physics of failure to PV systems, components, and materials, we are working to develop the framework of L\&DS, by developing metrics, metrology and tools to compare and quantify the response of PV systems, their components and materials to the wide variety of stressors they experience around the world, along with accelerated and multi-factor testing to determine PV lifetime. L\&DS requires the development of quantitative degradation mechanisms and rates for failure modes, so as to enable quantitative lifetime projections ${ }^{2}$. This paper is one step in developing this L\&DS framework for PV.

\section{Method}

As a new methodology for lifetime and degradation science for PV materials, components, modules and systems is developed, it is necessary to find a framework that can relate the mechanisms of degradation from multiple stressors to individual single and multi-factor tests in a way that is statistically significant, and perhaps predictive. Because it is often the synergistic effects of multiple stressors that do the most damage, a model that incorporates and correctly weights all stresses would be ideal. For many technology platforms, components that work well in one climatic region may fail rapidly in another due to this complex coupling of stressors and responses.

Stressors that impact PV materials and components can be characterized in terms of Instantaneous Stress Level $(\sigma)$, and net stress or Integrated Stress $(S)$, which is the Instantaneous Stress Level integrated over the length of time the stress was applied (Equation 1). Changing the Instantaneous Stress Level may change the material's response characteristics; therefore, stressors must be quantified in terms of both Instantaneous Stress Level and Integrated Stress (Equation 2).

$$
\begin{aligned}
& S=\int \sigma d t \\
& \sum S=\int\left(\sigma_{i} \otimes \sigma_{j} \ldots \otimes \sigma_{n}\right) d t
\end{aligned}
$$

A material's response (R) to both Instantaneous Stress Level and Integrated Stress may be a change in the spectral optical properties of a material, a loss of mechanical strength, or any change in properties arising due to stressors applied over time. As new ways are developed relating responses to stressors in PV, the general relationship between stress and response is a function of that stress (Equation 3):

$$
R=f\left({ }^{\sigma} S\right)=\int f(\sigma) \sigma d t
$$

If the stress response function, $f(\sigma)$, is assumed to be independent of integrated stress, Equation 3 can be rewritten (Equation 4):

$$
R=f(\sigma) \int \sigma d t
$$

Or, simplified (Equation 5):

$$
R=f(\sigma) S
$$

An expansion of this methodology to multiple stresses gives (Equation 6):

$$
R\left(S_{i}, S_{j}, \ldots S_{n}\right)=f\left(\sigma_{i}, \sigma_{j}, \ldots \sigma_{n}\right) \int\left(\sigma_{i} \otimes \sigma_{j} \ldots \otimes \sigma_{n}\right) d t
$$

where $S_{i}, S_{j}, \ldots S_{n}$ are different stresses that cause responses. The benefit of such a framework is that stresses can be separated and accounted for in this methodology. The convolution of these stresses implies that they can have synergistic effects, and the correction function, while unknown, depends on all stresses applied to the system. 


\section{Materials}

A proposed candidate material for back surface solar mirrors for MAPV systems is acrylic PMMA. PMMA is fairly durable, resistant to impact, has a low cost and is easily formable into complex shapes. Additionally, PMMA can be modified with additive packages in order to change its optical properties. Two formulated acrylic sheets are under investigation for use in MAPV systems. The first grade considered, UVR, is transparent to ultraviolet light and serves to produce a UV reflecting back surface solar mirror. The second grade considered is, MP, is a multipurpose PMMA formulation, which contains a light stabilizing additive package. Light stabilizer packages typically include UV absorbers and radical scavengers.

Several considerations must be taken into account when designing and constructing MAPV systems. While mirrors couple more light into PV modules, so that they produce more electricity, much of the infrared light incident on a solar cell cannot be transformed to energy, and is instead dissipated as heat. Increased thermal loads can cause early failure of solder joints, loss of mechanical properties of the encapsulant layer, or delamination of interfaces in the module. In addition to issues of thermal management, MAPV systems increase the ultraviolet load incident on the PV module. Ultraviolet light can induce photodarkening, decrease crosslinking, and cause chain scission reactions in polymers thus changing their physical properties and reducing their useful service life.

In most reflective CPV construction, especially medium and high CPV construction, front surface mirrors are used. While front surface mirrors have higher reflectance, they are prone to degradation if exposed to harsh weather conditions, and so require protection from the elements. Back surface mirrors are more durable in weather conditions, but suffer losses associated with internal refraction, and bulk absorbance (Figure 1). At the same time back surface mirrors use the optical absorption properties of the mirror substrate to spectrally tune the wavelength dependence of the mirrors reflectivity. This is the approach we use in MAPV with back surface mirrors. Different acrylic grades will produce different UV reflective mirrors.

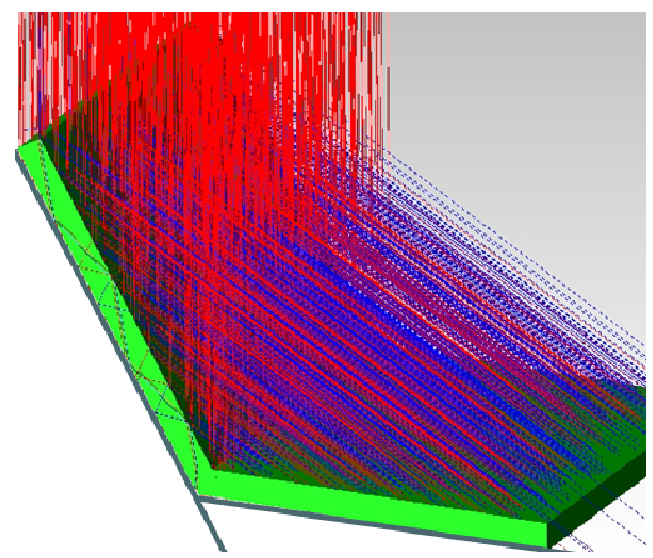

Figure 1: Typical back surface mirrors used in Mirror Augmentation of a flat panel PV module.

Durability of systems, components and materials is paramount for achieving a low levelized cost of energy with terrestrial photovoltaic systems. As such, investigation is underway to find and show mirror durability and lifetime performance, and through optimizing material choices, design systems that do not increase the power degradation rate of a solar panel. It is thought that by using formulated acrylic back surface mirrors, damaging ultraviolet light can be screened, preventing PV module degradation. 


\section{Methods:}

Parallel exposure of multiple samples on different platforms will enable cross correlation and data quality management and allow extraction of robust degradation mechanism and rate information from our L\&DS research.

Xenon arc exposures were performed on material coupons of one grade of PMMA ${ }^{3}$. A diverging continuous solar simulator allows for the exposure of material component sample with different levels of irradiance and the irradiance levels and cumulative dose can be quantitatively monitored using continuous power monitoring. If reciprocity can be shown, accelerated testing can give insights about expected results. If linearity over time can be shown, results can be extrapolated to make predictions about lifetime performance. Since the xenon source is filtered to match AM 1.5 radiation no consideration of additivity is required.

Accelerated UV exposures of two grades of acrylic were also performed in a QUV Weatherometer from Q-Labs. QUV instrumentation uses fluorescent lights that emit UV light. The phosphors in the fluorescent tubes can be designed to simulate different conditions. The bulbs used in the exposures were UVA-340 bulbs which emit radiation between 280 and $400 \mathrm{~nm}$. The UVA-340 spectrum is a good match to AM 1.5 in the UVA and B regions, where most damaging light exists in the solar spectrum ${ }^{4}$.

Typically, UV exposure rates are quantified as total UV or TUV (Equation 8). Because the spectra of AM 1.5 and UVA-340 are diverging significantly after $350 \mathrm{~nm}$, a cutoff point for UVA-340 has been arbitrarily established as $360 \mathrm{~nm}$.

$$
\begin{aligned}
& \text { TUV dose }=\iint_{280}^{400} A M 1.5 d \lambda d t \neq \iint_{280}^{400} U V A 340 d \lambda d t \\
& \text { SWUV dose }=\iint_{280}^{360} A M 1.5 d \lambda d t=\iint_{280}^{360} U V A 340 d \lambda d t
\end{aligned}
$$

We will refer to this damaging radiation as short-wave UV (SWUV) (Equation 9). PMMA acrylic is damaged by light between $260-320 \mathrm{~nm}$; therefore, this approximation is valid for comparing PMMA doses from different sources.

Exposures were carried out at $48.4 \mathrm{~kW} / \mathrm{m}^{2}$ for the AM 1.5 exposure for 18.26 days, which is a SWUV dose of $975.6 \mathrm{MJ} / \mathrm{m}^{2}$, and a TUV dose of $2623 \mathrm{MJ} / \mathrm{m}^{2}$. Exposure to UVA-340 light was $1.55 \mathrm{~W} / \mathrm{m}^{2} / \mathrm{nm}$ at $340 \mathrm{~nm}$ for a SWUV irradiance of $60.65 \mathrm{~W} / \mathrm{m}^{2}$. Exposure duration of 11.9 days gives a total SWUV dose of $62.0 \mathrm{MJ} / \mathrm{m}^{2}$. 

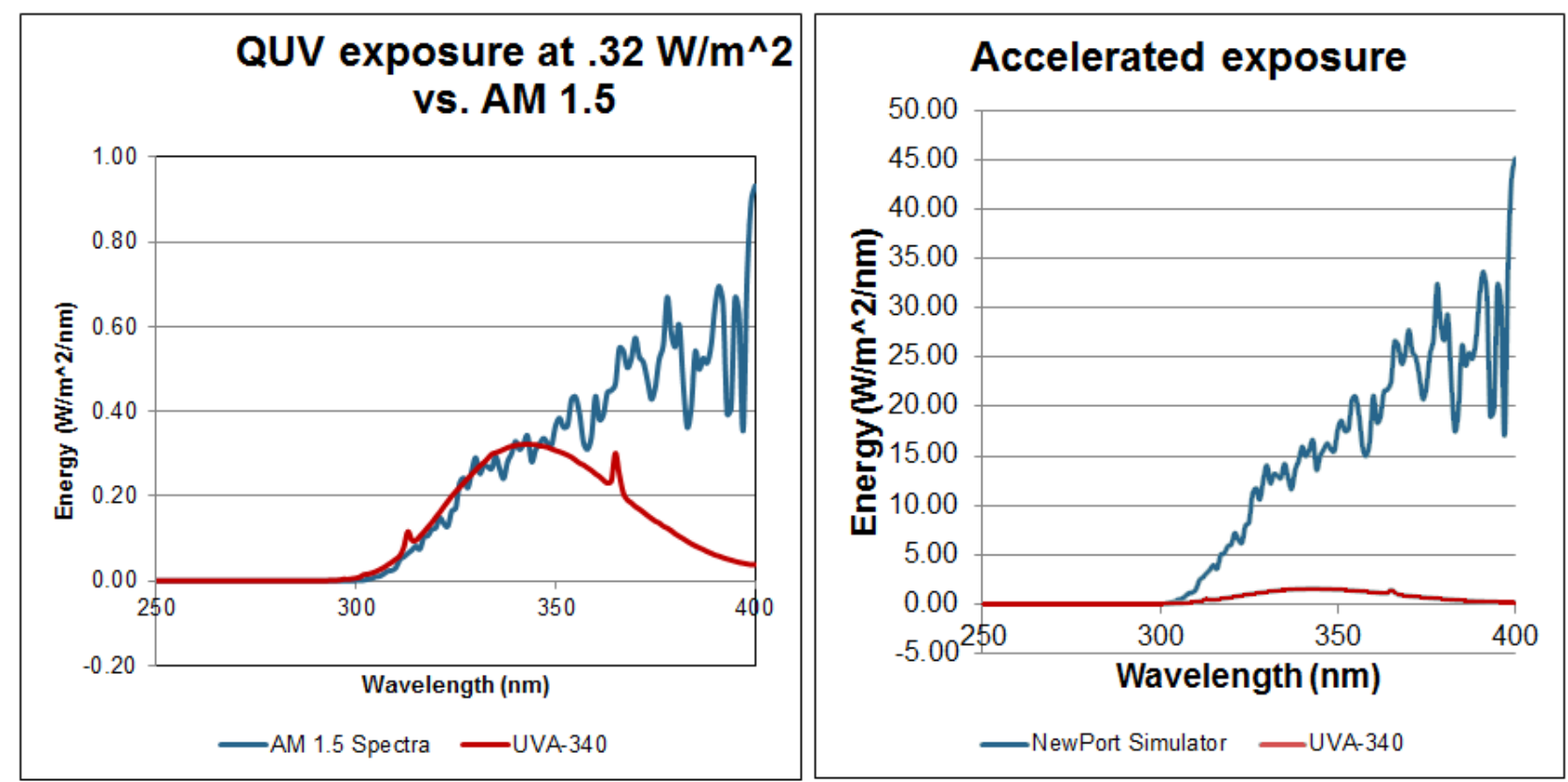

Figure 2: Comparison of (left) AM 1.5 at $1 \mathrm{~kW} / \mathrm{m}^{2}$ and UVA-340 exposure at $.32 \mathrm{~W} / \mathrm{m}^{2} / \mathrm{nm}$ at $340 \mathrm{~nm}$ and (right) the exposures performed here of $48.4 \mathrm{~kW} / \mathrm{m}^{2}$ and UVA-340 exposure at $1.55 \mathrm{~W} / \mathrm{m}^{2} / \mathrm{nm}$ at $340 \mathrm{~nm}$

\section{Results:}

Material response was characterized with optical instrumentation by looking at changes of the optical properties with SWUV exposure as the stressor. A metric under development, Induced Absorbance to Dose, calculated per unit of dose (either AM1.5 dose or SWUV dose) was calculated for the samples after exposure (Equation 10):

$$
\text { Incremental } \frac{A b s}{\mathrm{~cm}} \operatorname{per} \frac{G J}{\mathrm{~m}^{2}} \text { Dose } \equiv \frac{A b s_{i+1}(\lambda) / \mathrm{cm}^{-}-A b s_{i}(\lambda) / \mathrm{cm}}{\operatorname{Dose}_{i+1}-\text { Dose }_{i}}
$$



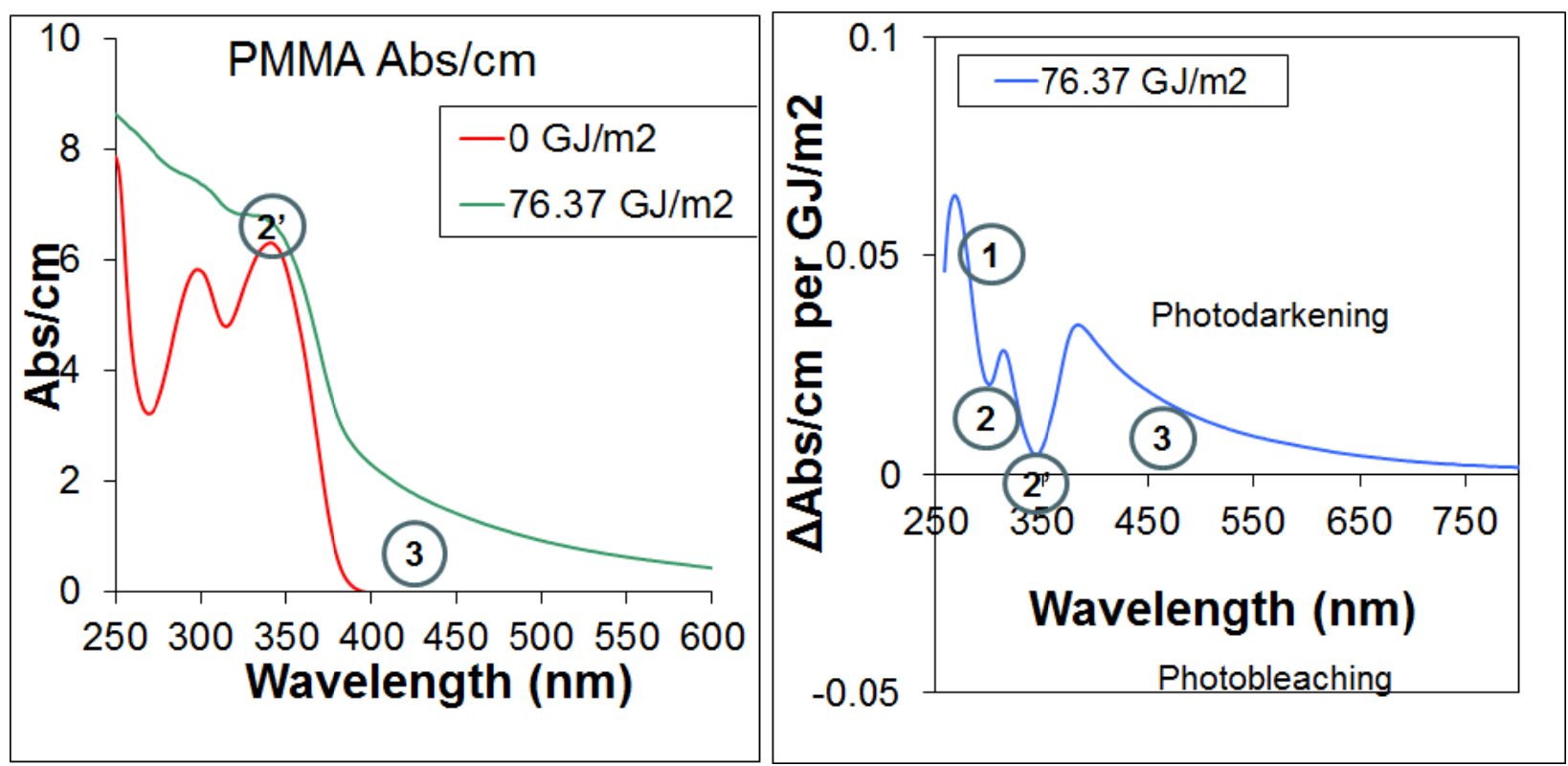

Figure 3: PMMA MP grade acrylic (left) before and after exposure to $48.4 \mathrm{~kW} / \mathrm{m}^{2}$ for $76.37 \mathrm{GJ} / \mathrm{m}^{2}$ total dose and (right) IAD at that point.
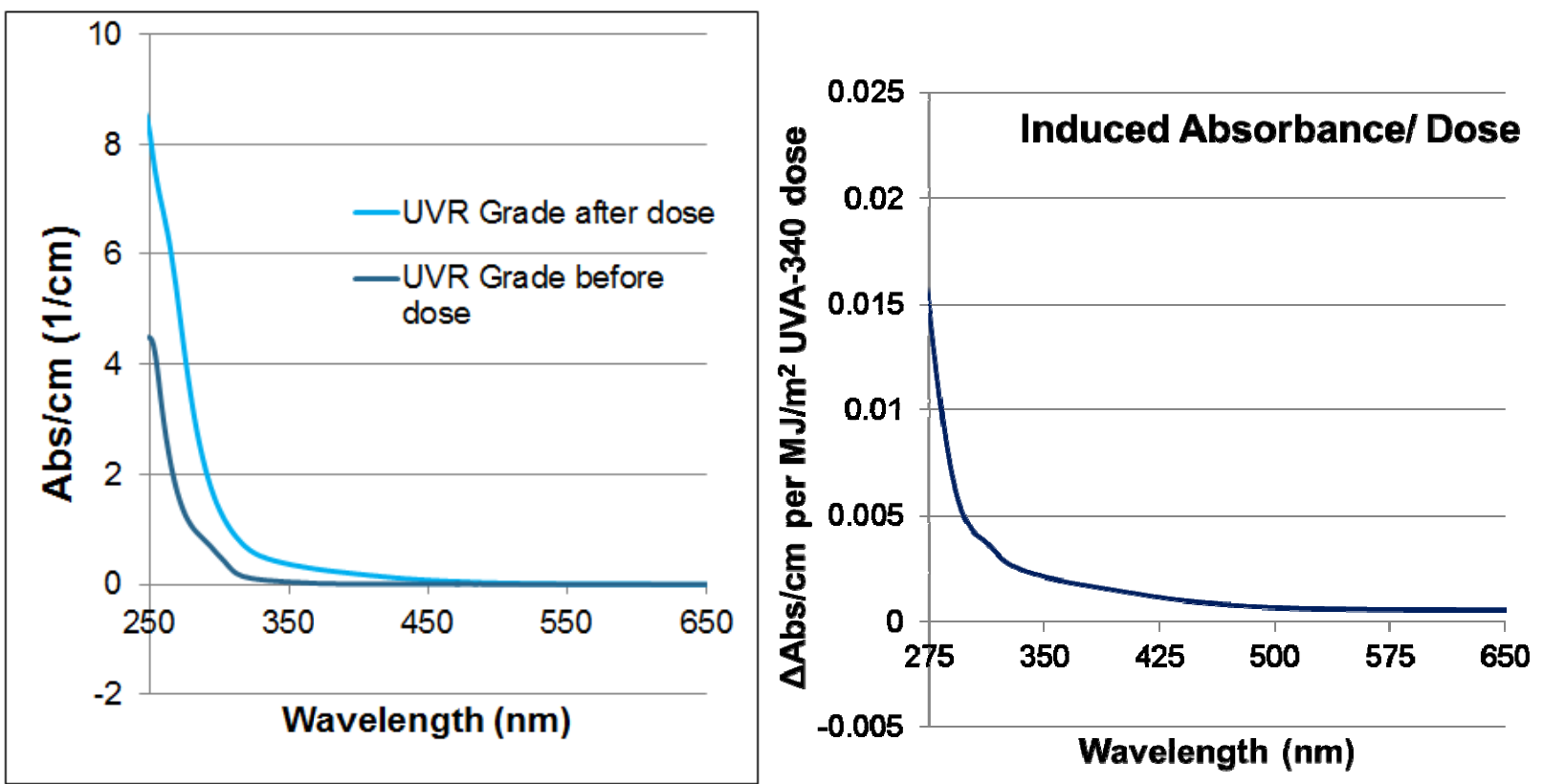

Figure 4: PMMA UVR grade acrylic (left) before and after exposure to UVA-340 for $62.0 \mathrm{MJ} / \mathrm{m}^{2}$ total dose and (right) IAD at that point. 

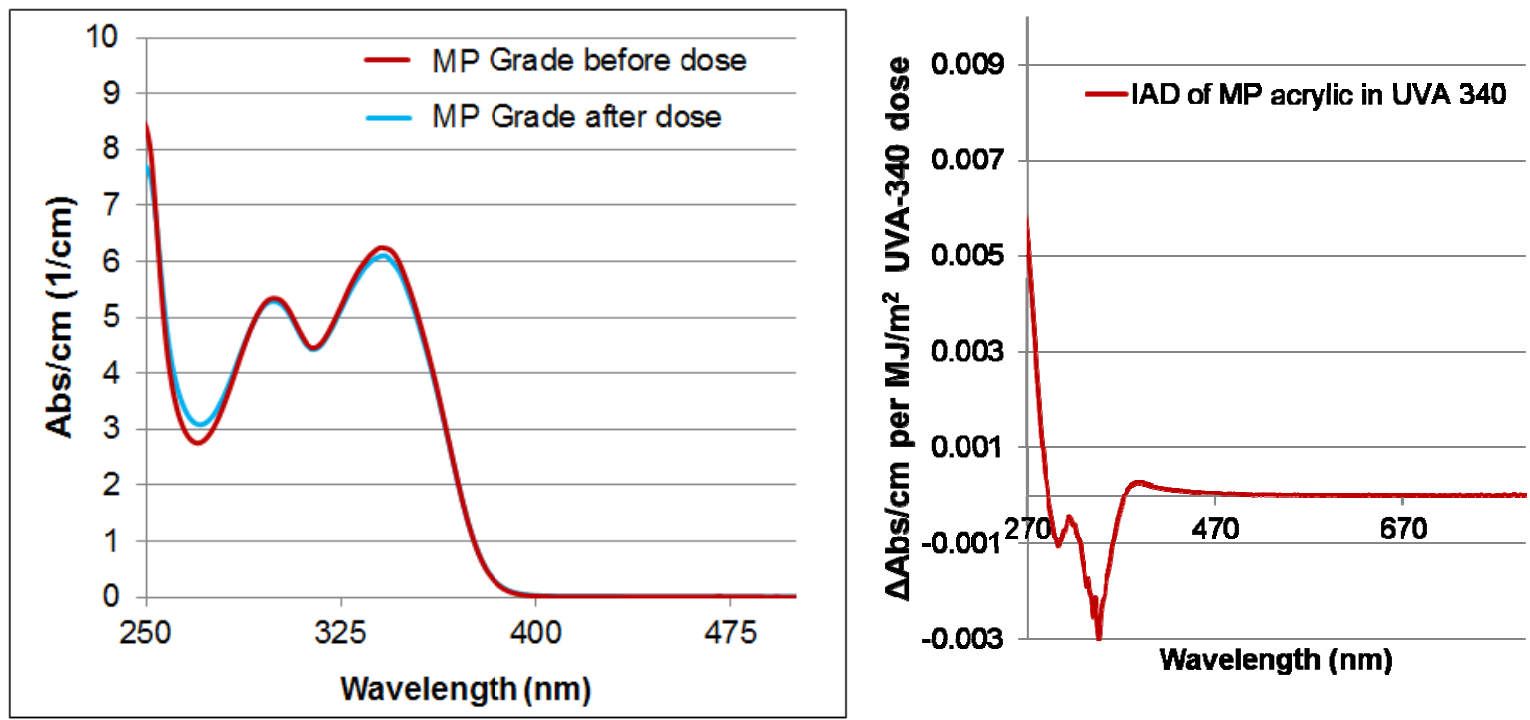

Figure 5: PMMA MP grade acrylic (left) before and after exposure to UVA-340 for $62.0 \mathrm{MJ} / \mathrm{m}^{2}$ total SWUV dose and (right) IAD at that point.

Another Metric that may be useful in quantifying damage to these types of materials is the ASTM yellowness index.

where $\mathrm{X}, \mathrm{Y}$ and $\mathrm{Z}$ are the CIE Tristimulus values. For D65/10 ${ }^{\circ} \mathrm{C}_{\mathrm{x}}=1.3013, \mathrm{C}_{\mathrm{z}}=1.1498$.

Yellowness index is a colorimetric measure of yellowing. Because YI is measured over a broad wavelength range it is more sensitive than typical spectral measurements.

\begin{tabular}{|c|c|}
\hline Sample & YI E313 [D65/10] \\
\hline UVR initial & 0.36 \\
\hline UVR final & 4.85 \\
\hline MP initial & 0.19 \\
\hline MP final & 0.56 \\
\hline
\end{tabular}

Table 1: YI for representative samples 

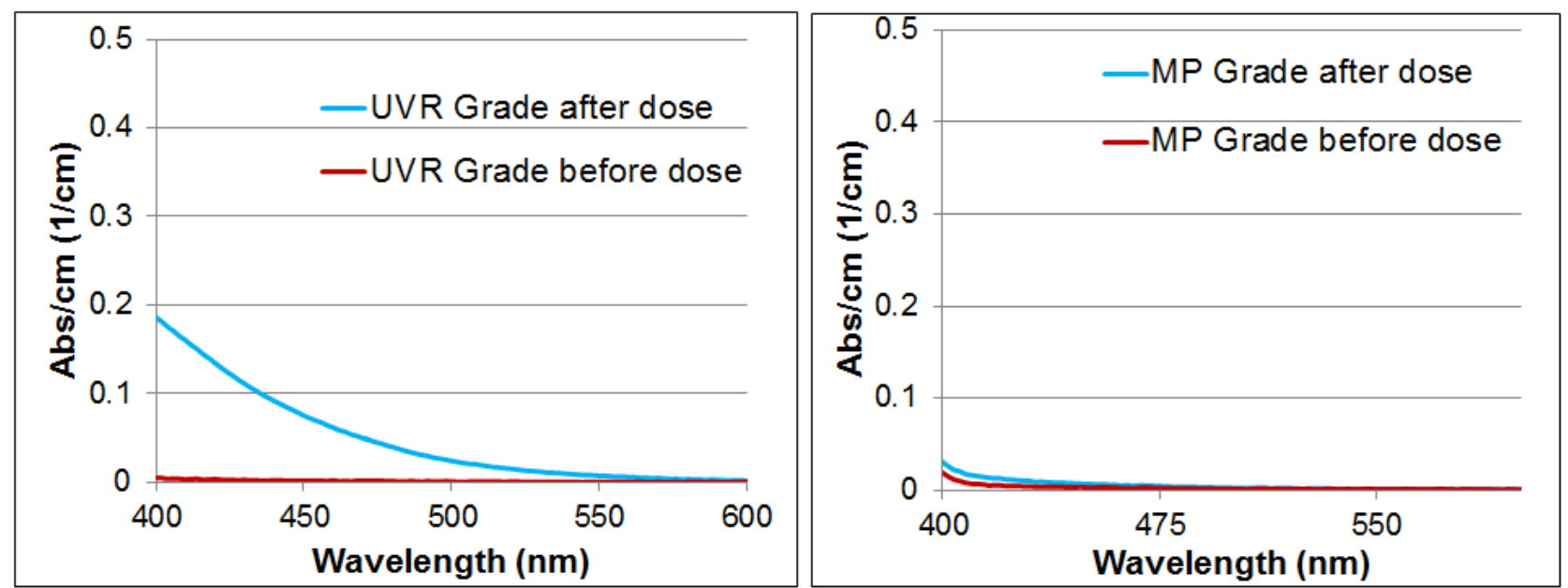

Figure 6:YI can be seen as the increased $\mathrm{Abs} / \mathrm{cm}$ of the samples post-exposure due to photodarkening in the visible region.

\section{Discussion:}

The instantaneous stress acceleration factor $\left(\mathrm{A}_{\text {irr }}\right)$ was 10.2 for the AM 1.5 exposure over the UVA-340 exposure using an SWUV irradiance basis. The integrated stress acceleration factor $\left(\mathrm{A}_{\text {dose }}\right)$ was 15.8 for the AM 1.5 exposure over the UVA-340 exposure (Figure 2). These acceleration factors bring these exposures into the Highly Accelerated Stress Test (HAST) regime where artifacts can arise due to the high Integrated Stress and/or Instantaneous Stress Levels. 


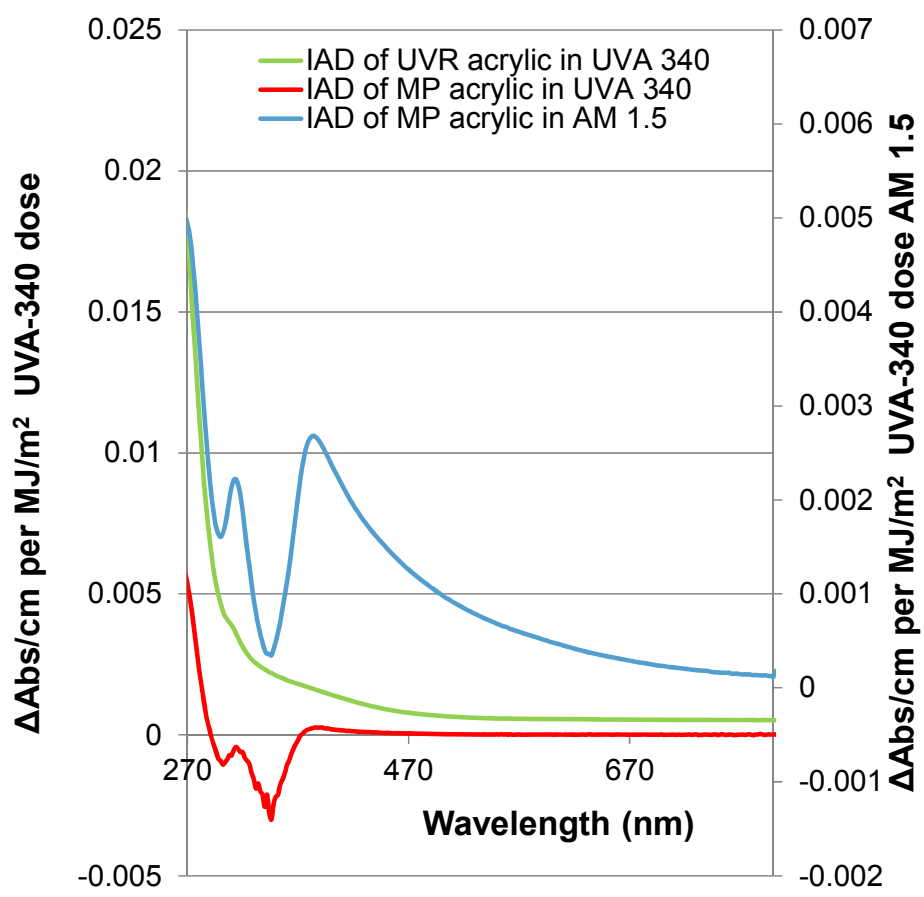

Figure 7: IAD comparison between grades and exposures

The graph of induced absorbance to dose shows that similar phenomena are observed in all samples and exposures in the region from $270-290 \mathrm{~nm}$ where photodarkening at the fundamental absorption edge is occurring (Figures 3-5). To a first order approximation, in the region from 270-290nm the response acceleration factor was 1, meaning that the same phenomena was present in the multipurpose acrylic for the first $62 \mathrm{MJ} / \mathrm{m}^{2} \mathrm{SWUV}$ dose from UVA-340 irradiation as over the $975.55 \mathrm{MJ} / \mathrm{m}^{2}$ dose of AM1.5 irradiation. In the region from 300-400nm, more experimentation is required in order to deconvolve degradation rates of the UV absorber package from the degradation of the base resin and its yellowing in the visible. The MP acrylic shows significant protection in UVA340 exposure as can be seen when comparing its IAD to the UVR grade. Increased yellowness was seen in all samples post exposure (Table 1). The increase of YI was dramatically induced in the MP acrylic through addition of UV absorbers ${ }^{5}$ (Figure 6).

Insight can be gained into the nature of the photodegradation mechanisms at play in the sample of MP acrylic exposed to AM 1.5 by inspection of its IAD curve (Figure 7). The valleys in the IAD curve of both MP exposures line up with the peaks of the UV absorber. This suggests that photobleaching of the additive package, their consumption or degradation and subsequent bulk polymer damage has occurred in the MP acrylic exposed to AM 1.5. It is also possible that damage was induced by light with $\lambda>400 \mathrm{~nm}$ in samples exposed to AM 1.5 radiation which is not present in the UVA-340 spectra.

\section{Conclusions:}

Samples of two types of acrylic PMMA were exposed to two stresses at two stress intensities for two doses in an effort to see the response of materials under different states of stress and after exposure to different amounts of 
total stress. All of the samples showed similarities in their degradation characteristics. Further study into degradation mechanisms will elucidate the exact phenomena that contribute to these material responses to stress.

It appears that materials additivity may hold true across the samples of PMMA containing UV stabilizers (MP) and not (UVR). The high dose exposure of MP grade acrylic appears to degrade the UV stabilizers, making it behave similar to UVR. This may be transferrable across multiple material systems exposed to different instantaneous stress levels and different integrated stresses.

Continuing work will require identification and quantization of stressors; identification of ways to meaningfully accelerate stresses and reproduce naturally occurring degradation mechanisms; application of this framework to other stressors such as temperature, humidity, mechanical loading, corrosive environments and applied loads; and deconvolution of the synergistic effects of multiple stressors. This will allow for the same framework to be applied to real world environmental conditions to enable indoor testing to be correlated to outdoor lifetimes.

\section{Acknowledgements}

The authors acknowledge funding from Ohio Third Frontier, Photovoltaics Program award Tech 11-060. In addition the assistance of Katie Groseclose, Dan Dryden and Laura S. Bruckman is appreciated.

\section{References:}

[1] U. S., D. O. E. Workshop on Science for Energy Technology workshop report, for DOE Basic Energy Science Advisory Committee, August 2010. http://science.energy.gov/ /media/bes/pdf/reports/files/setf rpt.pdf

[2] R. H. French, J. M. Rodríguez-Parada, M. K. Yang, R. A. Derryberry, N. T. Pfeiffenberger, “Optical Properties Of Polymeric Materials For Concentrator Photovoltaic Systems" Sol. Energy Mater. Sol. Cells, (2011),doi:10.1016/j.solmat.2011.02.025.

[3] Miller, D. C., Gedvilas, L. M., To, B., Kennedy, C.E. and Kurtz, S. R. , "Durability of Poly(Methyl Methacrylate) Lenses used in Concentrating Photovoltaic Modules," Proc. SPIE 7407, 74070G (2009)

[4] David C. Miller, Michael D. Kempe, Cheryl E. Kennedy, and Sarah R. Kurtz, "Analysis of transmitted optical spectrum enabling accelerated testing of multijunction concentrating photovoltaic designs", Opt. Eng. 50, 013003 (Jan 31, 2011); doi:10.1117/1.3530092

[5] Torikai, A., Hasegawa, H., "Wavelength effect on the accelerated photodegradation of polymethylmethacrylate," Polymer Degradation and Stability 61(1), 361-364 (1998). 